\title{
Féeries
}

Études sur le conte merveilleuX, XVII -XIX siècle

$12 \mid 2015$

À la croisée des genres

\section{Écrire sans les fées : naturalisme et merveilleux}

How to Write without the Fairys? Naturalism and Marvellous

\section{Chantal Pierre}

\section{(2) OpenEdition}

Journals

Édition électronique

URL : http://journals.openedition.org/feeries/968

ISSN : 1957-7753

Éditeur

UGA Éditions/Université Grenoble Alpes

Édition imprimée

Date de publication : 15 octobre 2015

Pagination : 93-107

ISBN : 978-2-84310-306-3

ISSN : 1766-2842

Référence électronique

Chantal Pierre, «Écrire sans les fées : naturalisme et merveilleux », Féeries [En ligne], 12 | 2015, mis en ligne le 15 octobre 2016, consulté le 08 septembre 2020. URL : http://journals.openedition.org/feeries/ 968

(c) Féeries 


\title{
Chantal Pierre
}

Université de Nantes / L'Amo (EA 4276)

\section{ÉCRIRE SANS LES FÉES : NATURALISME ET MERVEILLEUX}

\begin{abstract}
Il $y$ avait des petits enfants qui nous connaissaient par nos noms, nous aimaient, nous craignaient un peu; mais au lieu des beaux livres en or et en images, où ils apprenaient notre histoire, Paris leur a mis maintenant la science à la portée des enfants, de gros bouquins d'où l'ennui monte comme une poussière grise et efface dans les petits yeux nos palais enchantés et nos miroirs magiques. Alphonse Daudet, "Les fées de France», Contes du lundi, 1873 .
\end{abstract}

IL FAUT CHOISIR : ou la féerie ou la vie réelle» écrit Zola dans Le 《 Naturalisme au théâtre. Passant en revue les genres dramatiques du temps, le romancier naturaliste avoue sa «tendresse pour la féerie» dont le «charme est dans la franchise de la convention». La dernière apparition et le dernier succès de Nana sur scène a lieu d'ailleurs dans une féerie, Mélusine, où l'héroïne de Zola réalise «trois poses plastiques d'une fée muette et puissante ${ }^{2} »$. Ce genre, déclinant en cette fin de XIX ${ }^{\mathrm{e}}$ siècle mais qui connut un vif succès à l'époque romantique ${ }^{3}$, est «le seul cadre où

I. É. Zola, «Le Naturalisme au théâtre» (I88I), Euvres complètes, H. Mitterand (dir.), Paris, Nouveau Monde Éditions, 2002-20IO, t. IO, p. I8I. Les références aux écrits critiques de Zola seront faites dans cette édition.

2. É. Zola, Nana (i880), H. Mitterand (éd.), Les Rougon-Macquart, Paris, Gallimard, coll. «Bibliothèque de la Pléiade», 1960-I966, t. II, p. I47I. Les références aux Rougon-Macquart seront faites dans cette édition.

3. Sur l'essor du genre dramatique de la féerie, voir l'étude de R. Martin, La Féerie romantique sur les scènes parisiennes (I79I-I864), Paris, Honoré Champion, 2007. Rappelons que vingt ans durant, Flaubert a cherché à monter, en vain, une féerie, sentant disait-il un «revif» pour le genre (Correspondance, J. Bruneau (éd.), Paris, Gallimard, coll. «Bibliothèque de la Pléiade», I998, t. IV, 
[Zola] admet, au théâtre, le dédain du vrai ${ }^{4}$ " puisqu'il s'y trouve affiché, et par l'histoire, "peuplée de génies bienfaisants et de fées méchantes" ", et par la mise en scène, d'une "naïveté cherchée ${ }^{6}$ ". Zola oppose à ce genre, dont il aime la fantaisie et le carton-pâte, non pas le drame naturaliste dont il espère l'avènement, mais les comédies et les drames de son temps qui mentent "avec des ménagements infinis ${ }^{7}$ ", en s'appliquant à rendre vraisemblables des personnages et des situations impossibles. Car c'est ce domaine-là de la fiction, dramatique ou narrative, qui constitue sa véritable cible, et non pas le merveilleux, nullement réprouvé dans la mesure où l'invraisemblance est déclarée. Le «il était une fois» inaugural officialise le règne d'une imagination "qui se tient quitte de toute obligation" et «exhibe le comme $\mathrm{si}^{8}$ " de la fiction au lieu de le masquer. En ce sens, le conte merveilleux est une saine lecture ${ }^{9}$ contrairement aux romans " faux" dont le réalisme est dévoyé car, tout en s'efforçant de produire une image plausible du réel, ils racontent des histoires et proposent des personnages, "qui ne tiennent pas à la terre» comme dit souvent Zola, échappant aux déterminismes et à l'irréductible force des choses. C'est pourquoi, quand Zola se lance dans son roman Le Rêve (I888) - ce conte de fées à l'ombre d'une cathédrale - , écrit entre La Terre et La Bête humaine, il insiste dans son ébauche sur le pacte de lecture nouveau qu'il propose au lecteur des Rougon-Macquart: "Le Rêve serait le titre du volume, et c'est surtout ce qui me plaît. La vie telle qu'elle n'est pas, telle qu'on la rêve : tous bons, tous honnêtes, tous heureux ${ }^{10}$.» Le titre du roman tient lieu de «il était une fois», jouant franc-jeu comme un détrompe-l'œil situant le livre en marge de la production naturaliste ordinaire ${ }^{\mathrm{II}}$.

Il serait vain donc de chercher une querelle engagée par le naturalisme contre le merveilleux. En soi, le genre est considéré comme inoffensif

p. 465, Lettre à Ph. Leparfait, 3 février 1872). Pour écrire cette pièce qui deviendra Le Château des cours, l'auteur de Madame Bovary s'est plongé dans une longue lecture du Cabinet des fées.

4. É. Zola, «Le Naturalisme au théâtre», art. cité, p. I80.

5. Ibid.

6. Ibid., p. I8I.

7. Ibid.

8. M. Robert, Roman des origines et origines du roman, Paris, Gallimard, I972, p. 69.

9. Comme Flaubert (qui admirait l'énumération finale de Peau d'Âne), comme les Goncourt, Zola déclare aimer Perrault, pour «sa saveur de naïveté exquise» et avoue un faible particulier pour Le Chat Botté («Le Naturalisme au théâtre», art. cité, p. I82-I83).

Io. BNF, NAF, Ms io 323, fo 227.

II. Nous n'aborderons pas ce roman, à part dans la production zolienne, dont Marie Scarpa a bien montré les liens étroits avec Cendrillon dans L'Éternelle jeune fille, étude ethnocritique du Rêve, Paris, Honoré Champion, 2009. Voir aussi H. Pernoud, «Angélique au bois dormant, le conte de fées dans Le Rêve», Cahiers naturalistes, 2014, p. II5-I28. 
contrairement aux formes frauduleuses. Il serait plus juste de dire que ce sont les ennemis du naturalisme qui, catholiques en tête avec Barbey d'Aurevilly, formulent cette querelle, en accusant le naturalisme d'exterminer le merveilleux : en le chassant et du monde qu'il réduit à des principes matérialistes, et de la littérature en étendant sur elle son empire. Jean de Palacio a montré comment la réaction décadente passait justement par un retour au genre du conte de fées et par «une surenchère du merveilleux à la fin $\mathrm{du} \mathrm{XIX}{ }^{\mathrm{e}}$ et au tournant du $\mathrm{Xx}^{\mathrm{e}}$ siècle ${ }^{\mathrm{I2}} »$. En partie contemporaine du courant naturaliste, l'abondante féerie décadente recrée, a priori, un territoire sauvegardé, où fées et autres créatures merveilleuses peuvent revivre. Banville publie ses Contes féeriques chez Charpentier (éditeur des naturalistes) en I882 qui est aussi l'année de Pot-Bouille, le roman qui valut à Zola une infinité de caricatures le représentant avec un pot-de-chambre devenu emblématique. Ce retour en force s'effectue toutefois sur un mode suspect, celui justement de la "perversion» étudié par Palacio : Perrault, référence dominante du siècle en matière de féerie, subit détournements, parodies, suites détonantes qui exposent le merveilleux à des dérèglements destructeurs aboutissant à sa "désacralisation ${ }^{13}$ ". Ainsi la Décadence, à la fois, déplore la perte du merveilleux induit par l'hégémonie scientiste et - c'est sa perversité - produit et achève, de l'intérieur du genre, cette même perte. Avec son goût pour la déviance littéraire, le mouvement décadent a probablement plus saboté le conte de fées que le naturalisme, assurément moins pervers.

Disons-le : pour beaucoup de "petits naturalistes» qui évoluent dans l'entourage de Zola, comme Paul Alexis, Léon Hennique, Henry Céard, le merveilleux est tout simplement un non-problème, un horizon et un arrière-plan littéraires absents. Le genre ne fait trace et signe que pour les auteurs qui se sentent à l'étroit dans l'écriture documentaire. C'est alors que le texte naturaliste peut se tourner vers le merveilleux de deux façons : sous une forme "naïve», manifeste, à travers un métatexte féerique qui émerveille la réalité documentaire; ou sous une forme "mélancolique», plus implicite, qui met aux prises l'ancien et le moderne. La première manière est plutôt celle de Zola; la seconde, celle des Goncourt.

I2. J. de Palacio, Les Perversions du merveilleux, Ma mère l'Oye au tournant du siècle, [Paris], Séguier, 1993, p. 15.

13. Ibid., p. 258. Ainsi, dans «La Belle au bois rêvant" de Catulle Mendès (Les Oiseaux bleus, Paris, Havard, I888), la Belle réveillée reçoit fort mal le Prince, préférant se rendormir plutôt que de subir l'ennui de la vie conjugale. 


\section{Un naturalisme émerveillé}

Chez Zola, la féerie s'épanouit naturellement dans la métaphore et la comparaison par lesquelles Zola relance le genre dans les marges du récit naturaliste; il semble même que plus la modernité est avérée, plus la féerie se trouve sollicitée et affichée. Qu'il s'agisse d'une banque, d'un grand magasin, ou des Halles de Paris, le merveilleux s'invite dans les descriptions et produit le fameux «saut dans les étoiles ${ }^{14}$ " par lequel Zola définit son écriture. Ce trait d'écriture souvent commenté correspond à l'esprit de l'époque qui "veut donner droit de féerie à la science ${ }^{15}$ ", et inventera bientôt la "fée électricité», dépossédant le conte de son monopole du merveilleux. Zola féerise à coups d'images les emblèmes de la modernité. C'est ainsi que Denise écoute le récit de la fondation du Bonheur des dames "comme on écoute un conte de fées ${ }^{16}$ "; le magasin de nouveautés qui s'étend grossit à vue d'œil "pareil à l'ogre des contes ${ }^{17}$ » et l'on pourrait se demander si les devantures du magasin, où les mannequins ont la tête coupée, "remplacée par une grande étiquette piquée avec une épingle dans le molleton rouge du col ${ }^{18} "$, n'ont pas quelque chose à voir avec le cabinet sanglant de Barbe-Bleue; dans L’Argent (189I), les coffres de La Banque universelle sont comparés "aux tonneaux des contes, où dorment les trésors incalculables des fées ${ }^{19}$ "; dans Le Ventre de Paris (1873), les toits glacés des Halles les nuits d'hiver font songer Florent «au pays féerique des $\operatorname{contes}^{20}$ "; la Méchain, redoutable intrigante et usurière, que Saccard rencontre dans la rue le jour de l'ouverture de sa banque ressemble à «la fée mauvaise qui jette un sort sur les princesses au berceau ${ }^{21}$ "; Renée, figure des nuits parisiennes sous le Second Empire, s'apparente, dans La Curée (I87I), à une «fée excentrique des voluptés mondaines ${ }^{22}$ »... Dans

I4. "Je veux faire le saut dans les étoiles sur le tremplin de l'observation exacte" écrit Zola à son disciple Henry Céard (Correspondance, Presses de l'université de Montréal/Éditions du CNRS, I985, t. V, 22 mars I885, p. 249).

I5. J. Hetzel, Préface à Jean Macé, L'Arithmétique de grand-papa, histoire de deux petits marchands de pommes, Paris, Hetzel, 1863, p. 4.

I6. É. Zola, Au Bonheur des dames, Les Rougon-Macquart, éd. citée, t. III, p. 408.

17. "Maintenant, tel que le montrait la gravure des réclames, il s'était engraissé, pareil à l'ogre des contes, dont les épaules menacent de faire craquer les nuages.» (Ibid., p. 763) L'image publicitaire s'est ainsi substituée aux livres illustrés de contes de fées.

18. Ibid., p. 392.

19. É. Zola, L'Argent, Les Rougon-Macquart, éd. citée, t. V, p. 229.

20. "Les nuits limpides, les ruissellements de lune, l'emportaient dans le pays féerique des contes.»

(É. Zola, Le Ventre de Paris, Les Rougon-Macquart, éd. citée, t. I, p. 877)

2I. É. Zola, L’Argent, ouvr. cité, t. V, p. I38.

22. É. Zola, La Curée, Les Rougon-Macquart, éd. citée, t. I, p. 42I. 
La Conquête de Plassans (I874), les exemples de "folie lucide» empruntés au livre du $\mathrm{D}^{\mathrm{r}}$ Ulysse Trelat ${ }^{23}$ sont comparés à des contes de fées dont s'entretiennent, pour se divertir, les notables de Plassans :

L'histoire n'est pas très propre [...]. Le marquis d'une intelligence faible passait les journées entières dans son cabinet, où il se disait occupé à un grand ouvrage d'économie politique ... Au bout de dix ans, on y découvrit qu'il faisait de petites boulettes d'égale grosseur avec ....

- Avec ses excréments, acheva le docteur d'une voix si brave que le mot passa et ne fit même pas rougir les dames.

- Moi, dit l'abbé Bourrette, que ces anecdotes amusaient comme des contes de fées, j'ai eu une pénitente bien singulière... Elle avait la passion des mouches ${ }^{24}$.

Les récits de folie sont ainsi devenus le nouveau jeu mondain de ce $\mathrm{XIX}^{\mathrm{e}}$ siècle passionné d'aliénisme, comme le conte de fée était un divertissement des salons du XVII ${ }^{\mathrm{e}}$ siècle. Infinie, la chaîne analogique des substitutions aboutit ainsi à des configurations scabreuses dont il peut être difficile de définir assurément la fonction : penser la névropathie comme source d'un merveilleux moderne, la coprophilie figurant un nouveau prodige, discréditer des causeurs qui ne prennent pas le document scientifique au sérieux et le fictionnalisent, ironiser sur le merveilleux en lui faisant subir la proximité de la scatologie devenue un marqueur du naturalisme. Une chose est sûre, Zola féerise à tout-va les figures de la modernité, jusqu’à cette femme fugitive, cette passante baudelairienne, entrevue au petit matin dans les Halles :

Et comme ils étaient là, ils aperçurent une dame bien mise, pelotonnée d'un air de lassitude heureuse dans le coin d'un fiacre, perdu au milieu de l'encombrement de la chaussée, et filant sournoisement.

«C'est Cendrillon qui rentre sans ses pantoufles» dit Claude avec un sourire ${ }^{25}$.

Il ne s’agit pas de traces anecdotiques, de références décoratives, mais de signes qui apparient l'ancien et le nouveau.

23. La Folie lucide, étudiée et considérée au point de vue de la famille et de la société, Paris, A. Delahaye, I86I. Zola a résumé son livre dans des notes préparatoires (BNF, NAF Ms IO 294, fo I28-I3I) et le cite dans l'ébauche de La Faute de l'abbé Mouret: "Une jeune fille épouse un comte et le trouve faisant des boulettes avec ses excréments." ( $\mathrm{f}^{\circ} \mathrm{I}$ 28)

24 É. Zola, La Conquête de Plassans, Les Rougon-Macquart, éd. citée, t. I, p. II25.

25 É. Zola, Le Ventre de Paris, Les Rougon-Macquart, ouvr. cité, t. I, p. 623. 


\section{Désertion et nostalgie : le charme perdu des anciens contes}

Mais au fond, seul Zola est de ce naturalisme émerveillé, qui succombe au charme des inventions modernes. Car le naturalisme prend volontiers acte de son défaut de "charme» que Zola reconnaît ainsi sans ambages : "Certes aujourd'hui, nos romans n'ont plus le charme de Cendrillon et Barbe Bleue. Nous dressons simplement des procès verbaux et je comprends que les vieillards regrettent les contes dont on a bercé leur enfance ${ }^{26}$.» Les Goncourt, mais aussi Daudet avec eux, sont particulièrement sensibles à cette perte du merveilleux et familiers d'une mélancolie naturaliste qui, éprouvant la précarité des modernes, regrette le temps suspendu des contes. C'est pourquoi la figure de la Belle au bois dormant ${ }^{27}$ exerce une attraction remarquable sur ces écrivains qui se sont pensés comme des modernes : en ce siècle où "la forme d'une ville change plus vite, hélas, que le cœur d'un mortel ${ }^{28}$ ", l'enchantement de ce château figé cent ans durant et de cette princesse jeune et démodée à la fois forme l'exact contre-pied d'une modernité furieuse et d'une mode impérieuse, décrites par Zola dans $L a$ Curée (I87I) ou Au Bonheur des dames (I883), et par Edmond de Goncourt dans Chérie (1884), roman consacré en grande partie aux toilettes retentissantes portées par l'héroïne ${ }^{29}$. Dans la version de Perrault, la Princesse endormie se réveille habillée comme sa grand-mère, mais le Prince se garde bien de le lui dire ${ }^{30}$ — même s'il le remarque en son for intérieur,

26. É. Zola, «Documents littéraires», Euvres complètes, éd. citée, t. Io, p. 742.

27. Jean de Palacio remarque que ce conte est particulièrement prisé par les variations décadentes; innombrables sont les titres brodant sur le titre de Perrault : «La Belle au Bois dormait», "La Belle au bois rêvant», "La veuve au Bois dormant», «La Belle au Bois ... veillant...», «la Belle au lac dormant», «Belle au Bois d'Oubli dormant» (pour les références de ces textes, voir J. de Palacio, ouvr. cité, p. I42). Voir aussi l'article de Sophie Lucet, «Les Belles rendormies. Féeries fin-de-siècle » dans le présent volume.

28. Baudelaire, "Le Cygne», Les Fleurs du mal, Gallimard, coll. "Bibliothèque de la Pléiade», I975, p. 85 .

29. «Personne comme Chérie pour discerner, au premier coup d'œil, la disposition distinguée par excellence, personne pour adopter sentir et adopter l'originalité d'une coupe, personne pour découvrir le merveilleux petit chapeau sans apparence [...]» (E. de Goncourt, Chérie, J.-L. Cabanès et Ph. Hamon [éds], Paris, La Chasse au Snark, 2002, p. 238) Goncourt assimile même son personnage de victime de la mode à un "article-Paris", faisant de sa jeune fille une publicité vivante pour la mode parisienne. Sur la mode comme forme privilégiée de la modernité, voir R. Kopp, «Baudelaire : mode et modernité», Cahiers de l'Association internationale des études françaises, $\mathrm{n}^{\circ} 38$, 1986, p. 173-186.

30. «Le Prince aida la Princesse à se lever; elle était tout habillée et fort magnifiquement; mais il se garda bien de lui dire qu'elle était habillée comme sa mère-grand, et qu'elle avait un collet monté; elle n'en était pas moins belle. Ils passèrent dans un Salon de miroirs [...] les violons et les hautbois jouèrent de vieilles pièces, mais excellentes, quoiqu'il y eut près de cent ans qu'on ne les jouât plus.» (C. Perrault, Contes, J.-P. Collinet (éd.), Paris, Gallimard, coll. «Folio classique», 1999, 
signe de la sensibilité moderne et de la galanterie qui marque le genre au $\mathrm{XVII}^{\mathrm{e}}$ siècle. Le renouvellement de la garde-robe de la Belle au bois dormant peut donc encore attendre. La Princesse échappe ainsi à l'injonction d'être moderne; elle bénéficie du droit à être démodé, qu'une songerie des Goncourt évoque comme un soulagement :

J'ai toujours rêvé ceci, et ceci n'arrivera jamais. Je voudrais, la nuit, par une petite porte, à serrure rouillée, cachée dans un mur, je voudrais entrer dans un parc que je ne connaîtrais pas, un parc ombreux, mystérieux. Peu ou point de lune. Un petit pavillon, dedans une femme que je n'aurais jamais vue et qui ressemblerait à un portrait vu au musée. Un souper froid, une causerie où l'on ne parlerait d'aucune des choses du moment ni de l'année précédente. Un sourire de Belle au bois dormant, point de domestiques... Et s'en aller sans rien savoir, comme d'un bonheur, où on a été mené les yeux bandés, et ne pas même rechercher après, la femme, la maison, la porte, parce qu'il faut être discret avec un rêve... mais jamais, jamais, cela ne m’arrivera. Et cette idée me rend triste. (Journal, 28 décembre $1862^{31}$ )

La Belle au bois dormant rêvée par les Goncourt est une femme qui n'appartient pas à son siècle, icône intemporelle descendue d'un tableau et miraculeusement préservée, au fond de ce parc secret, loin de l'actualité immédiate et fugitive. La merveille tant désirée consiste à échapper à l'entêtant "universel reportage» de l'époque. Étrange vœu en définitive de la part d'auteurs qui consacreront une grande partie de leur temps à parler des faits et dits de leurs contemporains. En fait, ce songe en forme de conte de fée dessinant une uchronie bienheureuse apparaît comme une réaction au Journal qui voue les écrivains à la fatigue "des choses du moment et de l'année précédente ${ }^{32} »$. Quant à savoir si le songeur ne fait que causer avec la belle inconnue ou si les points de suspension du texte suggèrent une rencontre sexuelle indicible, il est difficile de trancher. Peu importe, car seule compte l'approche à la fois mystérieuse et familière

p. I3I) Sur le réveil de la belle endormie, voir M. Soriano qui fait de ce passage « une broderie pleine d'humour où l'auteur joue en quelque sorte à défendre les anciens contre les modernes" (Les Contes de Perrault, Paris, Gallimard, 1968, p. 13I).

3I. Journal, Mémoires de la vie littéraire, texte établi par R. Ricatte, Paris, Laffont, coll. «Bouquins», 1989, t. I, p. 909.

32. Dans Trente ans de Paris (Paris, Marpon-Flammarion, I888), Alphonse Daudet rapporte combien dans les années I86o il était charmé par la maison et la personne de Madame Ancelot, ancienne actrice de l'époque romantique, qui habitait dans une demeure de la rue Saint Guillaume, «oubliée d'Haussmann [qui] avait l'air endormi depuis des siècles sous la baguette d'un enchanteur» (p. 8I). L'hôtesse de ce salon littéraire s'apparente elle-même à une créature féerique : «Madame Ancelot, tout en blanc, rondelette et ridée comme une petite pomme rose, telle enfin qu'on se figure les fées des contes, qui ne peuvent mourir mais qui vieillissent pendant des mille ans. Madame Ancelot aimait les oiseaux, toujours comme les bonnes fées.» (Ibid., p. 8I) 
d'un espace-temps merveilleux, où l'échange de paroles est soustrait aux potins du jour.

Réalisant la merveille de la vie arrêtée, le conte de La Belle au bois dormant figure comme le plus antimoderne des contes, et, à ce titre, un de ceux qui parlent sans doute le plus aux hommes du XIX ${ }^{\mathrm{e}}$ siècle, à qui l'histoire a appris que s'arrêter, c'était mourir, qu'il s'agisse des aristocrates momifiés du Cabinet des Antiques de Balzac ou des commerçants «à l'ancienne» du Vieil Elbeuf dans Au Bonheur des dames de Zola. D'ailleurs, dans Les Rougon-Macquart, tous les personnages immobiles ou immobilisés sont condamnés par la lutte pour la vie qui règle l'évolution sociale. Nombreux sont les romans qui représentent un "coin" hors du temps, une demeure figée où dépérissent des êtres surannés, les vaincus de la vie moderne : la boutique du Vieil Elbeuf des Baudu, l'hôtel Béraud, dans La Curée, toute la ville endormie de Plassans à l'orée des Rougon-Macquart dans La Fortune des Rougon ${ }^{33}$ (I87I), ou encore la maison Beauvilliers dans L'Argent (189r). Dans ce roman, en face de l'immeuble où loge Saccard, deux femmes-cygnes mélancoliques, ultimes et dérisoires avatars des vieilles légendes celtiques et scandinaves ${ }^{34}$, vivent dans un jardin humide : une vieille femme et une jeune fille, qui se languit d'attendre un prince charmant qui ne viendra pas (elle a vingt-cinq ans, et donc a coiffé Sainte Catherine) :

Et dans cette paix humide de cave, en haut de ce perron disjoint, la première personne que Mme Caroline avait aperçue était la comtesse de Beauvilliers, une grande femme maigre de soixante ans, toute blanche, l'air noble, un peu surannée. Avec son grand nez droit, ses lèvres minces, son cou particulièrement long, elle avait l'air d'un cygne très ancien, d'une douceur désolée. Puis, derrière elle, presque aussitôt, s'était montrée sa fille, Alice de Beauvilliers, âgée de vingt cinq ans, mais si appauvrie qu'on l'aurait prise pour une fillette, sans le teint gâté et les traits déjà tirés du visage. C'était la mère encore $[\ldots]$ le cou allongé jusqu’à la disgrâce ${ }^{35}$.

Sortie de son domaine hors du temps, la jeune fille est violée dans un hôpital par un garçon hirsute, véritable bête humaine, qui n'est autre que le fils du banquier... Les appétits déchaînés de la chair et de l'argent abîment et anéantissent la fragile Alice de Beauvilliers. C'est que, chez Zola,

33. La cité provençale est entourée «de fortifications, mangées de lierre et couvertes de giroflées sauvages, tout au plus égales en hauteur et en épaisseur aux murailles d'un couvent» et ferme ses portes à double tour le soir (É. Zola, La Fortune des Rougon, Les Rougon-Macquart, éd. citée, t. I, p. 38).

34. Sur ces figures mythiques, voir H. Heine, De L'Allemagne, Euvres complètes, Paris, MichelLévy, I855, vol. 2, septième partie.

35. É. Zola, L’Argent, ouvr. cité, p. 68. 
les habitants de ces lieux anachroniques ignorent la violence et le désordre des relations sociales et interindividuelles. Attachés aux normes et aux rites d'une société traditionnelle en passe de disparaître, ils subissent de plein fouet cette «bousculade des appétits ${ }^{36}$ " qui, pour Zola, caractérise la société issue de la Révolution. Or, le conte ordonne la bousculade, en la ritualisant, comme le montrent les études de la psychanalyse ou celles de l'anthropologie culturelle. À ce titre, depuis Bruno Bettelheim, les cent ans de sommeil infligés à la princesse représentent la patience à laquelle doit se soumettre la sexualité de la jeune fille. Quoique pubère, celle-ci doit passer par une phase de latence ou une épreuve, qui different la rencontre sexuelle. Le discours de la psychanalyse est corroboré par celui de l'anthropologie : les contes merveilleux relatent ainsi les rites de passages, fondés sur la triade séparation, marge, agrégation ${ }^{37}$, qui mènent à la maturité sexuelle et l'intégration sociale dans les sociétés traditionnelles. L'urbanisation, la prolétarisation de populations venues des campagnes, la mobilité sociale et géographique fragilisent ces rites de passage. Or, les deux héroïnes emblématiques du naturalisme, Gervaise Macquart et Germinie Lacerteux, sont justement, à l'origine, des déracinées du XIX ${ }^{\mathrm{e}}$ siècle, villageoises jetées sur le pavé parisien, qui regrettent le coin de rue et le village de leur enfance. Élisa, la prostituée du roman d'Edmond de Goncourt, a vécu son enfance dans les Vosges dont elle se souvient aux dernières heures de sa vie en prison; sa mémoire retrouve toutes les coutumes d'une enfance villageoise et catholique ${ }^{38}$ : la pêche aux écrevisses dans les eaux claires des ruisseaux ${ }^{39}$, les pèlerinages à la Vierge, les veillées où l'on jouait, Noël où elle recevait "un quenieu, gâteau à cinq cornes, que donnent ce jour, dans les Vosges, les parrains à leurs filleules ", la fête du village où elle était parée de larges rubans appelés des «liasses», et, surtout, les cerisiers en fleurs comparés à

36. La formule est fondatrice pour l'œuvre de Zola; elle figure dans les «Notes générales sur la marche de l'œuvre", texte préparatoire à la série : «Mon roman eût été impossible avant 89 . Je le base donc sur la vérité du temps, la bousculade des ambitions et des appétits. [...] Le moment est trouble. C'est le trouble du moment que je peins." ("Documents et plans préparatoires», Les Rougon-Macquart, éd. citée, t. V, p. 1738-1739)

37. Formulée par Arnold van Gennep dans ses travaux sur le folklore (Le Folklore, croyances et coutumes populaires françaises, Paris, Stock, 1924). Voir, par exemple, sur cette articulation du conte et des rites de passage, A. Monjaret, «De l'épingle à l'aiguille», L'Homme, revue de l'EHESS, $\mathrm{n}^{\circ}$ 173, 2005. Voir également le livre de M. Scarpa consacré au Rêve de Zola, L'Éternelle jeune fille: une ethnocritique du Rêve de Zola (ouvr. cité).

38. E. de Goncourt, La Fille Élisa [1877], D. Baguley (éd.), Paris, Honoré Champion, 20ıo, p. $225-228$.

39. Par contraste, à son arrivée à Paris, l'enfant est frappée par les eaux troubles de la Marne, s'écriant "Oh! Comme il a dû pleuvoir ici!» (ibid., p. 225). 
de «blanches fleurs d'oranger» dont les pétales tombaient sur elle comme sur une mariée encore vierge... Au terme d'une vie à la fois chaotique et répétitive, où Élisa passe de bordel en bordel, l'héroïne de Goncourt se trouve gratifiée, rétrospectivement, d'une enfance ritualisée, placée sous la double protection d'un parrain bienveillant et de la Vierge, très proche de la fée dans la dévotion catholique populaire. Rien d'étonnant alors à ce que la petite Élisa s'ensevelisse et s'endorme sous les fleurs virginales des cerisiers, petite Belle au bois dormant préraphaélite du Val d'Ajol, tentée par un engourdissement et une immobilité bienheureuse. Venue à Paris, la petite fille est projetée dans un vrai cauchemar, une «abominable vie», traumatisée par les accouchements et les avortements pratiqués par sa mère sage-femme, côtoyant les rudes réalités de la sexualité avant qu'elle ne choisisse finalement de se prostituer. Avant le roman naturaliste d'Élisa, qui la conduit de la prostitution à la folie, il y a eu un récit d'enfance, malheureusement interrompu, procédant de l'enfance du récit. C'est ainsi que s'esquisse le conte d'une petite fille tentée par la chair et désireuse de rester vierge en dormant sous les fleurs. La fin du roman d'Edmond de Goncourt porte donc à son comble la nostalgie du conte au sein du roman naturaliste, en énumérant, à l'issue de ce roman moderne, les traditions populaires d'une campagne qui a marqué l'enfance des deux frères, et en recomposant au sein de cette vie champêtre l'image fantasmatique de la Belle au bois dormant.

Ainsi, si Le Rêve de Zola est l'histoire d'une jeune fille qui s'extrait d'un sordide roman naturaliste pour aborder au domaine merveilleux du conte de fées, le roman naturaliste non seulement passe par l'éviction de la féerie - cela va sans dire —, mais surtout tend à inscrire, en creux, les traces de ce récit absent et de ce monde révolu. La destinée de Chérie, dernière hérö̈ne goncourtienne, la plus parisienne et la plus sophistiquée des jeunes filles à la mode, dépend de cette perte. Par toute une série d'infractions et de manques, elle se soustrait en effet aux épreuves de maturation et d'intégration, qui lui auraient permis de devenir adulte, épouse et bourgeoise accomplie dans la société du Second Empire. Histoire d'une jeune fille qui ne se marie pas et qui en meurt, Chérie est certes un roman de l'hystérie qui constitue, comme cela va de soi, la motivation naturaliste explicite et développée de cet échec. Mais autre chose se dessine et se joue à l'arrièreplan du roman et de la scène parisienne où il se déroule. Avant Paris et la vie mondaine, Chérie a habité un pays qui s'apparente à celui des contes : dans un château sis sur les ruines d'un ancien couvent, entouré de forêts et de ravins, la petite fille, orpheline de père et séparée de sa mère aimante, vivait et grandissait avec son grand-père Maréchal de France, vieux bonhomme très épris de sa petite-fille... On reconnaît sans peine 
certains invariants du récit féerique. Il y a même, dans les marges de ce domaine au nom virginal («Nonains-le-Muguet»), des figures protectrices et initiatrices, Lizadie la vieille nourrice conteuse et Mascaro le garçon des bois, qui vit «dans une ancienne loge de portier en ruine, près d'une porte abandonnée ${ }^{40}$ ", à la lisière du domaine. Mais ni l'une ni l'autre n'entraînent Chérie au-delà des limites du "parc merveilleux ${ }^{4 \mathrm{I}}$ " pour la séparer de ce grand-père trop aimant. Nulle séparation à endurer, nulle peau d'âne éprouvante à enfiler pour Chérie afin d'atteindre sa maturité. Au contraire, suivant à Paris son grand-père (qui renvoie Lizadie, jugée trop paysanne), elle entre à quinze ans avec frénésie dans la vie mondaine : Chérie soumet ainsi à son caprice et le temps, quand, rentrée trop tard du bal, elle retarde la pendule de son grand-père ${ }^{42}$, et les codes vestimentaires quand elle obtient "de s'habiller comme une femme mariée ${ }^{43}$ ", et les prétendants qu'elle refuse les uns après les autres. De même, tous les objets se multiplient comme dans un conte devenu fou : les souliers de bal ${ }^{44}$, les robes merveilleuses, les parfums formant une "vie à outrance ${ }^{45}$ " dont le propre est d'ignorer la patience et la mesure du rite, et d'ainsi décomposer l'unité ${ }^{46}$ que le conte tend au contraire à produire.

À force de sorties toujours recommencées, Chérie s'épuise et finit par se replier dans ses pensées sur "un coin de Lorraine, une habitation perdue dans de vieux arbres, un peuple d'animaux domestiques, grouillant et tapageant autour de ses jupes, une vie de nature et de campagne ${ }^{47}$ ». Elle en regrette les «eaux vives» et les légendes entendues enfant autour de la Fontaine-au-Chêne ${ }^{48}$. En ce dernier roman consacré aux infimes détails de la sociabilité élégante, l'écrivain reconstitue paradoxalement un domaine dédié à une littérature ancienne et populaire, où il fait mourir et reposer son héroïne hystérique. L'auteur prend même la peine (ou plutôt le plaisir) de faire écouter intégralement à son lecteur un conte merveilleux du folk-

40. E. de Goncourt, Chérie, ouvr. cité, p. 98.

4I. Ibid., p. 80.

42. Ibid., p. 225.

43. Ibid., p. 224.

44. «Par terre, dans un coin, s'élevait un amoncellement de souliers de satin blanc, essayés et jetés les uns sur les autres, et au milieu s'en trouvaient des paires qui n'avaient même pas été sorties de leur coquette enveloppe de toile grise, aux initiales brodées : tous ces souliers répandant une forte senteur de peau d'Espagne dont ils étaient parfumés. " (Ibid., p. 294)

45. L'expression est de Zola à propos de Renée dans La Curée (ouvr. cité, t. I, p. 334).

46. Décomposition de l'unité (sociale et stylistique) dont on sait qu'elle est l'essence de la Décadence pour Paul Bourget (Essais de psychologie contemporaine, Paris, Gallimard, 1993 [1885], p. I4).

47. Ibid., p. 283.

48. Ibid., p. 232-233. 
lore lorrain raconté par la vieille Lizadie ${ }^{49}$ : trois villageois de Ruaux-lesFous, patoisants et simples d'esprit, vont à la ville pour la première fois "afin d'y apprendre le français ${ }^{50}$ "; ils se font emprisonner parce qu'ils ne connaissent que trois expressions de l'idiome national et sont finalement sauvés puis ramenés chez eux par des fées qui protègent les habitants de ce village. C'est l'histoire édifiante du péril qu'il y a à être moderne, et du bonheur qu'il y a à rester chez soi et à cultiver son patois. Chérie, dernier roman du dernier des Goncourt, restitue ainsi, sans la pervertir, une belle histoire de fées et de simples d'esprit racontée simplement par une vieille femme à une petite fille. La modernité naturaliste portée à son paroxysme se donne le plaisir simple du merveilleux et de l'oralité.

\section{Où sont les enfants?}

Les romantiques, dans leur quête de ressourcement, l'ont abondamment dit et théorisé : la nostalgie du merveilleux est celle, confondue, d'une enfance de la littérature et d'une littérature de l'enfance ${ }^{\text {st }}$. Le naturalisme s'impose, au contraire, comme entrée dans l'âge adulte de la littérature : lucide, clinique, démythifiante et résolument contemporaine. De ce tournant, les enfants sont les premières «victimes». À deux titres : d'une part, parce qu'avec ses innombrables enfants vicieux, dégénérés, trop vite et mal grandis (du voyeurisme de la petite Nana observant les ébats de Gervaise et Lantier à la "maturité hâtive ${ }^{52}$ " de Chérie en passant par Maxime, le "petit crevé" de La Curée), le mouvement dégrade la représentation de l'enfance; d'autre part, parce que le naturalisme semble vouloir inventer une nouvelle espèce d'enfant, réformé et réinventé par ses lectures. Le combat naturaliste, tel que Zola l'envisage du moins, est en effet total et passe par une mobilisation éducative. Cela implique une discrimination entre bons et mauvais livres, et la prescription de ceux qu'il faut lire et de ce qu'il vaut mieux ne pas lire. Ennemis des fictions mensongères mais vraisemblables, les écrivains souhaitent inventer un nouveau lecteur, et

49. Ibid., p. IO2-IO5. Le village des Ruaux est situé à proximité de Plombières, station thermale vosgienne de renom où se rendait l'Impératrice. Il est mentionné par les guides touristiques du Second Empire; on trouve ainsi l'histoire des fées de Ruaux rapportée dans Le Tour du Monde, nouveau journal des voyages, Paris, Hachette, I867, vol. I. Le conte de Lizadie est reproduit dans le présent volume, accompagné d'une notice sur ses sources folkloriques.

50. Ibid., p. I02.

5I. Sur ce point, voir C. Millet, Le Légendaire au XIX siècle, Paris, PUF, 1997, p. 2I-30.

52. Chérie, ouvr. cité, p. 262. 
surtout une nouvelle lectrice, dont on sait les inquiétudes qu'elle ne cesse de susciter depuis les origines du roman. Les Goncourt et Zola sont persuadés que la plupart des jeunes filles se sont perdues par la lecture de romances sentimentales :

Mon frère et moi, nous le disons quelque part, le livre obscène, le livre érotique, n'a aucune action sur la jeune fille française. Quand elle arrive à se perdre par la lecture, elle se perd par un livre sentimental, par un livre chastement romanesque. [...] il lui faut dans le livre qu'elle tient entre ses mains de l'amour qui se passe en des milieux autres que nos salons, de l'amour qui ait même un certain recul dans le passé, de l'amour traversé de malheurs, de péripéties, de fatalités — de l'amour entouré d'accessoires, qui en font une sorte de sentiment idéal, et sans copie de ce qu'elle a sous les yeux ${ }^{53}$.

Tirant les conséquences de ce constat désastreux qu'il partage ${ }^{54}$, Zola imagine dans La Joie de vivre (I884) une jeune fille exemplaire, modèle d'auto-apprentissage naturaliste, qui appréhende le monde à travers les ouvrages de science et dédaigne toute forme de fiction, Robinson ou les contes de fées.

[...] c'était sur des ouvrages de médecine laissés dans l'armoire, qu'elle passait des journées entières, les yeux élargis par le besoin d'apprendre [...] elle les sortait dès que sa tante tournait le dos, puis les replaçait au moindre bruit, sans hâte, non pas en curieuse coupable, mais en travailleuse dont les parents auraient contrarié la vocation. [...] elle s'était acharnée sur l'anatomie descriptive, avant de passer au Traité de physiologie. Alors, cette enfant apprit comme dans un devoir ce que l'on cache aux vierges jusqu'à la nuit des noces. Elle feuilletait les planches de l'Anatomie, ces planches superbes d'une réalité saignante; elle s'arrêtait à chacun des organes, pénétrait les plus secrets dont on avait fait la honte de l'homme et de la femme [...] La découverte lente de cette machine humaine l'emplissait d'admiration. Elle lisait cela passionnément, jamais les contes de fées, ni Robinson, autrefois ne lui avaient ainsi élargi l'intelligencess.

La solution zolienne qui consiste à substituer à la lecture des fictions la lecture des planches d'anatomie est radicale et évidemment caricaturale. Elle alimente une peur sourde qui se traduit par un scénario catastrophiste partagé et répété par les auteurs de la seconde moitié du siècle. Nombreux sont ceux qui, en réaction au programme de lecture zolien, craignent l'apparition d'une nouvelle espèce d'enfants : savants et incrédules. En

53. Ibid., p. 159.

54. "C'est l'idéal qui jette la jeune fille aux bras du passant, c'est l'idéal qui fait la femme adultère [...]. Les corrupteurs sont les idéalistes qui mentent." (É. Zola, Le Roman expérimental, CEuvres complètes, éd. citée, t. 9, p. 369).

55. É. Zola, La Joie de vivre, Les Rougon-Macquart, éd. citée, t. III, p. 854. 
I885, dans Le Livre de mon ami, Anatole France, un peu excédé, accuse ainsi l'édition de participer à cette entreprise d'éradication du merveilleux dans l'esprit des enfants. Il s'en prend tout particulièrement à l'infatigable apôtre de la vulgarisation scientifique, Louis Figuier :

Les catalogues illustrés des livres d'étrennes enfantines présentent aux yeux, pour les séduire, des crabes, des araignées, des nids de chenille, des appareils à gaz. C'est à décourager d'être enfant. À chaque fin d'année, les traités de vulgarisation scientifique, innombrables comme les lames de l'Océan, inondent et submergent nos familles [...] On m'a montré hier l'Alphabet des Merveilles de l'Industrie! Dans dix ans, nous serons tous électriciens! M. Louis Figuier [...] sort de sa placidité ordinaire à la seule pensée que les petits garçons et les petites filles de France peuvent connaître encore Peau d'Âne. Il a composé une préface exprès pour dire aux parents de retirer à leurs enfants les contes de Perrault et de les remplacer par les ouvrages du Dr. Ludovicus Ficus son ami ${ }^{i 6}$.

Edmond de Goncourt, prophète d'apocalypse, de renchérir : ces jeunes mutants, victimes de l'hégémonie scientiste, seront responsables à terme de la mort des contes de fées. Déplorant que les nouvelles générations d'enfants soient plus intéressés par la chimie et la physique que par les contes de fées ou par Robinson, l'écrivain en déduit carrément la fin de la littérature et de l'art, comme dans cette note du Journal de I882 :

Au milieu de la conversation des grandes personnes, j'entends un gamin dire à un autre gamin qui dîne à côté de lui : «Mais la densité de l'eau?» Voici la génération présente des enfants. Ils ne sont plus amusés, ils ne sont plus intéressés que par des joujoux scientifiques, que par de la chimie ou de la physique à portée de leur petite cervelle. Les contes de fées ou les Robinson ne leur parlent plus. C'est là, je crois, un symptôme de la mort de la littérature et de l'art chez les hommes du xx siècles7.

Pour Goncourt, qui rejoint par là le romantisme et sa mythologie du ressourcement, l'essence de la création littéraire et artistique réside dans cette "poésie naturelle» (Mme De Staël) du merveilleux qui ramène aux

56. A. France, «La bibliothèque de Suzanne», Le Livre de mon ami, Paris, Calmann-Lévy, 1923 [I885], p. 26I-262. Louvrage se termine sur un dialogue entre trois amis autour de l'origine et du sens des contes de fées. Rappelons que Jules Hetzel, une vingtaine d'années plus tôt, avait édité en I86I, pour les étrennes justement, les Contes de Perrault, illustrés par Gustave Doré.

57. Journal, ouvr. cité, t. II, is juillet i882, p. 949. Voir cette autre remarque furibonde du même Edmond de Goncourt : «On se demandait dans un coin de notre table de Brébant, comment on pourrait remplacer, plus tard, dans la cervelle française les choses poétiques, idéales, surnaturelles : la partie chimérique que met dans l'enfance une légende de saint, un conte de fée. De sa rue voix de gendarme du matérialisme, Charles Robin s'est écrié : "On y mettra de l'Homère!"

Non, très illustre micrographe, un chant de l'lliade ne parlera pas à l'intelligence de l'enfant, comme lui parle une histoire bêtement merveilleuse de vieille femme, de nourrice.» (Ibid., 22 janvier 1878 , p. 765 ) 
premières émotions de la vie. Si ce sens de la poésie n'est plus transmis aux enfants, parce qu'ils auront été très tôt formés aux sciences, c'est le processus même de la création littéraire qui est atteint. Zola, qui ne doute pas de la compatibilité des sciences et de la littérature et qui pratique un naturalisme émerveillé, n'a pas de ces frayeurs anticipatrices et ne prédit ni l'extinction de l'enfance ni celle de la littérature. Car, fort de sa vision spontanément et grossièrement merveilleuse du monde moderne, où le grand magasin est un ogre et la banque une grotte aux fées, l'auteur des Rougon-Macquart s'invente en "primitif", rendu à une enfance de l'humanité qui ignore le sentiment de la fin et de l'impuissance. Les Goncourt, et a fortiori Edmond écrivain solitaire et endeuillé, ignorent ce rapport enchanté à leur siècle, ce qui explique, pour partie, la crainte de l'impuissance qui court dans une œuvre, davantage portée à la collation et la collection de documents qu'à la fabulation ${ }^{58}$. C'est pourquoi le regret des contes du temps passé ne se limite pas seulement à la nostalgie passéiste d'une société stable et structurée, mais il implique chez eux la peur fondamentale d'avoir perdu, à force de notes documentaires prises sur le vif, d'enquêtes et d'indiscrétions, le naïf secret de la poésie, détenu par la vieille Lizadie.

A priori coupé des sources du conte, car sûr de ses propres sources, la science et le document ${ }^{59}$, le roman naturaliste, dans ce qu'il a de meilleur, n'oublie cependant pas le merveilleux que les romantiques ont pensé comme origine de la poésie, et que les décadents ont regretté en le défigurant. Zola, qui peint «le trouble du moment ${ }^{60}$ ", peuple la vie moderne de fées, d'ogres et de croquemitaines, esquissant un récit merveilleux qui s'allie, sans s'y opposer, au "procès-verbal ». Goncourt, aux avant-postes de la modernité, en expérimente la frénésie et le vide : il lui oppose, sans illusion, par bribes isolées, le retour au rite et à l'oralité du conte, dont il sait la littérature dessaisie.

58. La "pensée de collection» des Goncourt étudiée par Dominique Pety est en effet taxée de stérilité. Voir Les Goncourt et la collection, Paris, Droz, 2003, p. 25 I.

59. Edmond de Goncourt le répète encore dans la préface des Frères Zemganno : «Seuls, disons-le bien haut, les documents humains font les bons livres : les livres où il y a de la vraie humanité sur ses jambes.» (Paris, Charpentier, I879, p. X)

6o. É. Zola, «Notes générales sur la marche de l'œuvre», Les Rougon-Macquart, éd. citée, t. V, p. 1739 . 



\begin{abstract}
ANNEXE
"LE CONTE DE LIZADIE»

EDMOND DE GONCOURT, CHÉRIE,

CHAPITRE XV, PARIS, CHARPENTIER, 1884
\end{abstract}

RESTE ENCORE UN PEU, ma petite Chérie, je te conterai une belle histoire... Tu écoutes?»

C'était la vieille Lizadie qui parlait à l'enfant qu'elle avait placée sur les genoux de sa mère et que la folle tenait, comme par obéissance, entre des bras inertes.

«Ils étaient trois hommes qui marchaient à la file l'un de l'autre à travers les champs.

C'étaient le maire, l'adjoint, le maître d'école.

Au village d'où ils venaient, on ne causait que le patois, et ils s'étaient mis en route pour la ville afin d'y apprendre le français.

Et tous les trois, midi sonnant, savaient déjà une phrase qu'ils avaient entendue comme ça, sur le chemin.

Entre nous tous, répétait entre ses dents le maire.

Pour de la toile, répétait l'adjoint.

Comme de raison, répétait le maître d'école.

Si bien qu'en ragotant ainsi ils arrivaient dans un bois où il y avait un pendu. Pendant qu'ils regardaient la grimace que, dans un grand arbre, faisait le branché, voici des gendarmes en tournée qui leur demandent si c'étaient pas eux qui l'auraient accroché là-haut, et le maître d'école de répondre aussi tôt : "Comme de raison"; l'adjoint : "Pour de la toile»; le maire : «Entre nous tous».

Là-dessus le maire, l'adjoint, le maître d'école sont emmenés par les gendarmes en prison.»

Les yeux de la folle descendaient parfois sur la petite fille placée sur ses genoux, s'y arrêtaient un moment avec une attention où il semblait y avoir l'effort de se ressouvenir, puis s'en détournaient comme d'une chose indifférente et se remettaient à regarder dans le vide. 
«Faut dire, reprenait Lizadie, que le maire, l'adjoint, le maitre d'école étaient des hommes du village de Ruaux-les-Fous, ainsi appelé à cause de la simplesse d'esprit des gens, mais qui pour cela ont été de tout temps protégés par les fées dont le château se voit encore à côté... un tout petit château, au milieu du Fays, et qui est en forme d'un 8, mais qui n'a jamais été fini. À ce propos, on raconte que les fées, qui n'avaient qu'une nuit pour le bâtir, se sont amusées dans le bois à cueillir des brimbelles ${ }^{\mathrm{I}}$, puis qu'elles ont été surprises par le jour avant de l'avoir achevé. Il se répète encore qu'il y a dessous des souterrains fermés par de grosses pierres qu'on n'ose pas déplacer, parce que la croyance est chez nous qu'ils sont pleins de serpents-volants que des enfants de Claire-Fontaine ont vus, chacun avec un diamant planté sur la tête.

Donc les trois hommes de Ruaux étaient bien tristes entre les quatre murs de la prison... lorsque, attendez voir... il entrait dans leur cachot une bonne petite fée du Fays... une fée reconnaissable à sa robe noire, moirée de feux follets. Bon, la fée s'approche du maire en train d'allumer sa pipe avec une allumette qui ne prenait pas... Et voyez-vous la petite curieuse qui voudrait bien savoir ce qui va arriver!... Or voilà qu'avec le bout de l'allumette... où c'est du phosphore, la bonne petite fée trace sur la muraille noire un grand cheval, dont le tracé se met à éclairer comme un ver luisant... et, bonté divine, ça devient dans la minute un cheval vivant... un cheval de feu... sur lequel la fée saute, légère comme la plume, en faisant signe aux trois hommes de monter derrière elle; - même que le pauvre maître d'école, qui n'avait pas trouvé place dessus, était obligé de se pendre des deux mains à la queue du cheval, d'où trissait ${ }^{2}$ tout plein de flammèches et d'étincelles... Mais, Seigneur Dieu! étaient-ils déjà assez haut dans le ciel sur ce cheval qui, trip, trep, trip, trep, trip, trep, allait cent fois plus vite qu'un chemin de fer!... Ils étaient si haut que les arbres, les maisons, les bêtes, ne leur paraissaient pas plus grands que ceux de la botte d'amusettes qui est là... et quand ils étaient en haut comme ça, ne voilà-t-il pas qu'ils se sentent redescendre, redescendre à grand $p_{i t a t a^{3}} \ldots$ oh! mais vite, vite, plus vite encore que jamais oiseau de

I. Nom donné aux myrtilles dans l'Est de la France, et notamment dans les Vosges.

2. Trisser : gicler, jaillir avec force. Le terme est répertorié par Jean Daunay dans Parlers de Champagne : pour un classement thématique du vocabulaire des anciens parlers de Champagne (Aube, Marne, Haute-Marne), Langres-Saints-Geosmes, Éditions Dominique Guéniot, 1998, p. 238.

3. Cette onomatopée qui imite le galop du cheval est signalée par Jean Daunay, ibid., p. 2 I4. 
l'air n'a volé... oui, ils se sentent redescendre vers une grande bande bleue, qui leur fait crier, tout tremoulants ${ }^{4}$; la mer! la mer!

Patatra! le derrière par terre... sur une ruade du cheval de feu, une ruade plus forte que le vent le plus fou, les trois hommes sont jetés, la tête en bas, dans ce qu'ils croyaient la mer... et qui était un champ de lin... qu'on sait être tout bleu... et d'un bleu de bluet... quand le lin est en fleur. Et après qu'ils avaient marché une heure dans le champ bleu, les trois hommes étaient pas mal ébaubis d'apercevoir le clocher de Ruaux, où ils arrivaient presque aussitôt et où ils mouraient bien des années après, n’ayant, jusqu'au jour de leur mort, jamais plus parlé que patois.»

C'était par des histoires semblables que la vieille Lizadie, espérant toujours une résurrection de la maternité chez sa maîtresse, retenait quelques instants près de cette maman l'enfant ennuyée et désireuse de s'en aller.

\section{AUX SOURCES DU CONTE DE LIZADIE}

Nous proposons ici quelques hypothèses sur les sources et origines de l'histoire racontée par Lizadie à la petite Chérie, en présence de sa mère devenue folle. Il est possible que certaines séquences proviennent de Pélagie Denis, gouvernante d'Edmond de Goncourt dans les années I870 et 1880 (on la trouve régulièrement citée dans le Journal); celle-ci est en effet née et enterrée à Ruaux, petite localité située près de Plombières 5 , dans cette partie sud des Vosges que les Goncourt connaissaient bien (Élisa, le personnage du roman La Fille Élisa, vient du Val d'Ajol, non loin de là). Par ailleurs, la légende du château figure dans de nombreux textes érudits d'antiquaires locaux qui s'interrogent notamment sur les origines druidiques du lieu; elle est passée dans les guides touristiques et

4. Étrangement, ce mot n'appartient nullement au patois lorrain ni champenois ou vosgien. Il s'agit d'un verbe provençal, très proche du verbe français " trémuler » (trembler) ; les Goncourt soulignent cependant cette forme inhabituelle.

5. Sur Pélagie et Blanche Denis, sa fille, voir le livre de Marcel Valzer, Viens à Ruaux, en passant par Plombières, F. Lallemand, 1984. Je remercie chaleureusement M. Régis Robert pour ses avis éclairés et pour tous les documents qu'il m’a transmis ou signalés. Toutes mes recherches sur ce passage de Chérie lui sont redevables. 
des journaux à grande diffusion ${ }^{6}$, dont les Goncourt ont pu avoir facilement connaissance.

L'histoire est composée de deux récits, l'un enchâssant l'autre. Elle associe, d'une part, le récit des mésaventures des trois habitants de Ruaux, et, d'autre part, le récit de la construction du château par les fées qui vient s'intercaler dans le précédent. Dans toutes les sources que nous avons pu consulter, nous ne trouvons jamais intégralement l'histoire de Lizadie, mais seulement des éléments épars qui sont de deux ordres : des éléments relevant du conte drolatique qui relate, en série, les aventures burlesques et mémorables d'une localité peuplée de simples d'esprit agissant en dépit du bon sens ${ }^{7}$; des éléments relevant de la légende merveilleuse qui attribue à l'action des fées une curiosité locale (un château en ruines). Voici, par exemple, ce qu'on pouvait lire dans le Guide Joanne de 1867 :

Ruaux était autrefois une ville, et cette ville n'était habitée que par des fous. Les fées qui, tout le monde sait cela, sont des créatures excellentes, couvraient d'une protection particulière cette population d'insensés. Aussi, elles avaient résolu de bâtir un château fort, destiné à préserver de toute attaque le village des innocents. L'emplacement qu'elles avaient choisi était une éminence située dans la forêt dite Fays. Le château devait être bâti en une nuit. Mais, ou la besogne était plus longue que ces bonnes ouvrières l'avaient cru, ou quelque mauvais génie avait des motifs pour s'opposer à ce que Ruaux fût fortifié, car les fées furent surprises par les premiers rayons de l'aurore, avant qu'elles eussent achevé leur ouvrage. Artistes de nuit, elles ne pouvaient finir le travail du moment que le jour paraissait, elles laissèrent tomber les pierres qu'elles portaient dans les plis de leurs robes noires, tachetées de feux follets, et elles disparurent ${ }^{8}$.

Le guide touristique relève la double spécificité, drolatique et féerique, de la localité, conforme à la tradition populaire pour laquelle l'innocent est un esprit "fée» (nommé «fada» en provençal). Touché par les fées, celui-ci est réputé bénéficier de leur protection. Mais, dans les histoires de fous de Ruaux collectées par les folkloristes et parvenues jusqu'à nous, nos recherches n'ont pas permis de retrouver l'épisode de la mésaventure lin-

6. Voir Le Tour du Monde, «Nouveau journal des voyages», I867, premier semestre, Hachette, p. 34I et suiv.

7. Par exemple celle que rappelle Léopold-François Sauvé dans Le Folklore des Hautes-Vosges, Paris, Maisonneuve, I889 : "Il est juste de citer en premier lieu les Fous de Ruaux, canton de Plombières, auxquels on prête généralement tous les traits de la sottise qui court le monde. En voici quelques-uns : un étranger - un baigneur, sans doute, de Plombières - a satisfait à un besoin naturel auprès de leur église : ils décident de la déplacer pour que le contact de l'odorant présent ne la souille pas davantage.» (p. 25I) D'innombrables variations existent sur ce canevas.

8. Plombières, itinéraire descriptif et médical, par MM. Lemoine et L'Héritier, Paris, Librairie Hachette, collection des Guides Joanne, 1867, p. 195-196. 
guistique survenue au maire, à l'adjoint et au maître d'école. En revanche, le scénario figure presque à l'identique dans un conte de Fraimbois, autre village lorrain, situé au sud de Nancy, qui a vu se développer, comme à Ruaux, toute une tradition orale moquant la bêtise de ses habitants ${ }^{9}$, en l'occurrence, dans le conte $\mathrm{n}^{\circ} 27$, celle de trois villageois ayant décidé d'apprendre le français à la ville. En voici le texte :

- Nous sommes tout de même fort bêtes, nous devrions aller un peu à Nancy pour apprendre à parler le français.

[Les trois Fraimboisiens se retrouvent à Nancy où a lieu le certificat d'études. Dans une auberge, ils entendent parler des maîtres d'école.]

Mes trois benêts tendaient l'oreille. Mais comme ils avaient une courte mémoire, le premier n'a retenu que les mots suivants : «nous tous»; le deuxième : "pour de l'argent"; le troisième : "Comme de juste».

Ils n'en avaient jamais entendu autant, ce qui a fait qu'ils se croyaient tout savants. [Sur le chemin du retour, ils trouvent sur le corps mort d'un homme étranglé. Les gendarmes passent.]

- Qui est-ce qui a tué cet homme-là?

- Nous tous, répond le premier.

- Pourquoi l'avez-vous tué? qu'ils redemandent en tirant les menottes.

- Pour de l'argent, répond le deuxième.

- Eh bien, nous allons tous vous mettre en prison, se met à hurler le garde-champêtre.

- Comme de juste, dit le troisième.

[Présentés au tribunal, les trois Fraimboisiens sont reconnus par le juge qui les innocente.]

- Demeurez à Fraimbois pour surveiller vos cacattes de femmes qui se sont peut-être donné du bon temps, tandis que vous étiez à Nancy comme trois pauvres foutues bêtes, pour apprendre le beau langage.

On peut légitimement penser que Goncourt, qui est né à Nancy, connaissait cette histoire, grâce au lien qu'il avait gardé avec la culture populaire lorraine, grâce peut-être aussi à sa gouvernante, Pélagie Denis. Mais, venu de Ruaux, de Fraimbois ou d'ailleurs, le récit des trois benêts patoisants connait des modifications et variations notables dans la version de Lizadie, la conteuse d'Edmond de Goncourt. Car dans les histoires drôles de Fraimbois, comme dans celles de Ruaux que nous avons pu lire, les fées et les phénomènes merveilleux sont cruellement absents. Les épisodes développent un comique fondé sur le grotesque et l'absurde, tandis que le dénouement consiste à confirmer l'insondable stupidité des personnages. Nulle trace de merveilleux en effet dans le dénouement du conte $\mathrm{n}^{\circ} 27$ Fraimbois puisque les trois malheureux patoisants sont sauvés par un juge

9. Ces contes étaient très en vogue à la fin du XIX ${ }^{\mathrm{e}}$ siècle, publiés en patois sous forme de cartes postales par un instituteur, Athanase Grandjacquot. Ils ont été rassemblés et traduits par Jean Lanher, Les Contes de Fraimbois, Nancy, Presses Universitaires de Nancy, 1984. 
qui les identifie et en profite pour se moquer d'eux. Rien de tel dans la version de Lizadie. La vieille bonne, qui s'adresse à une petite fille, sollicite un merveilleux spectaculaire pour dénouer le tragique quiproquo. Apparaît la personne d'une «bonne petite fée» dans le cachot des trois fous où elle fait surgir, avec une allumette devenue baguette magique, un cheval de feu. Les fous s'envolent alors sur son dos, montent très haut dans le ciel avant d'être "parachutés» et de regagner enfin leur village. Cette irruption providentielle d'une féerie pleine d'effets spéciaux protège effectivement les innocents de Ruaux des sarcasmes ironiques et humiliants qu'inflige le juge de la ville aux prévenus de Fraimbois; ce sont ainsi les railleries d'une modernité incrédule et triomphante que les fabuleux prodiges de la fée épargnent aux fous de Ruaux. En réenchantant ces fous qui sont des marginaux et des inadaptés, la version de Lizadie se fait bienveillante.

Chantal Pierre 